\title{
Interactive comment on "The stable carbon isotope signature of methane produced by saprotrophic fungi”' by Moritz Schroll et al.
}

\section{Moritz Schroll et al.}

moritz.schroll@geow.uni-heidelberg.de

Received and published: 5 June 2020

We thank Lukas Kohl for the positive evaluation of our work and for the helpful comments to improve the manuscript. All comments and requested changes were taken into account. Please note that comments by the referee are in italics and that in the authors' answer the mentioned line numbers refer to the version of the revised manuscript including track changes.

Referee 1: Schroll and co-authors studied the stable carbon isotope values of methane emitted during the aerobic decomposition of organic matter by two fungal species. Methane production by fungi during plant litter decomposition is a novel pathway of methane formation, that was recently documented by the authors and others. This

Printer-friendly version

Discussion paper 
manuscript, however, is the first study of the stable carbon isotope (d13C) values associated with this novel pathway and their relationship substrate $\mathrm{d} 13 \mathrm{C}$ values. This study addresses/closes a knowledge gap in the isotope systematics of atmospheric methane that is relevant to the Biogeosciences readership. The authors used state of the art methods, and their conclusions are well supported by their results. The manuscript is well structured and easy to follow. The study's strength is that this is the first study of its kind and provides unique stable isotope fractionation factors between biomass and methane produced by fungi. The study also used very robust measurement methods (GC/IRMS with preconcentration) that exceeds the precision, accuracy, and specificity of laser-based analysers. The main limitations of the study are that the authors did not test for contaminations by other microbial species during this study (this was, however, tested by the authors in similar incubations in a previous study). Another limitation is that the authors were not able to identify controls over relatively large variations in methane isotope values beside differences between $\mathrm{C} 3$ and $\mathrm{C} 4$ plants. This, however, is understandable given that the biochemistry of aerobic methane production in fungi remains poorly understood, and the authors contribution will surely help elucidate these pathways in the future. Authors: We thank the referee for the positive evaluation of our manuscript. The reviewer's concerns are addressed below.

\section{Main comment:}

The authors used two distinct fungal species, and state that these species include both white rot and brown rot fungi. However, I was unable to find where in the manuscript the authors identify which fungal species belongs to which group.

Authors: A description of which fungal species belongs to white and brown rot fungi was added to section '2.1 Selected fungi' (L77-78).

Printer-friendly version

Minor comments:

1) L56. remove 'applications of' for easier sentence structure 
Authors: Change applied.

2) L57-58. clarify what 'they' refers to in 'they might be used.., also, avoid 'fingerprints' ('characteristic d13C values?)

Authors: 'they' was clarified as ' $\delta 13 \mathrm{C}-\mathrm{CH} 4$ values' and 'fingerprints' was changed to 'characteristic $\delta 13 \mathrm{C}$ values'.

3) L61. 'global isotopic patters': Do you mean the d13C values of atmospheric CH4?'

Authors: Correct. For clarification purposes ' $\delta 13 \mathrm{C}-\mathrm{CH} 4$ ' was added.

4) L66. 'isotope patterns': stable isotope values?

Authors: Change applied.

5) L77-78. clarify which fungi is the white rot and which one is the brown rot one.

Authors: A specification of which fungal species belongs to white and brown rot fungi was added to section '2.1 Selected fungi'.

6) L148. is $0.06 \mathrm{mg}$ correct? This seems a very low sample inweight for EA/IRMS, although not impossible. Also, did you analyse analytical replicates? A single $0.06 \mathrm{mg}$ inweight is likely associated with a significant subsampling error.

Authors: Yes, the sample weight is correct. Around $0.06 \mathrm{mg}$ of sample was used for the EA/IRMS measurements. Three replicates of each substrate were measured $(n=3)$. Standard deviations for $\delta 13 \mathrm{C}$ of the substrates were $0.5 \%$ for pine wood, $0.6 \%$ for grass and $0.1 \%$ for corn.

7) L170-173. You could add a note that the low R2 resulted from the lack of a change in $\mathrm{d} 13 \mathrm{C}$ values (emission d13C was similar to background $\mathrm{d} 13 \mathrm{C}$ ). In this case, a low $\mathrm{R} 2$ does not indicate a poor relation between concentration and $\mathrm{d} 13 \mathrm{C}$ value.

Authors: Thank you for the very helpful comment. We added a note according to the reviewer's suggestion.

Printer-friendly version

Discussion paper 
8) L176-177. 'The SDs are given with a confidence interval of $1 \sigma$ ': sentence not needed and meaningless.

Authors: Change applied.

9) L182-188. not needed, can be removed.

Authors: Please note, that for better readability we would like to keep this paragraph as it clearly explains the structure of section ' 3 Results and Discussion' and makes this section easy to follow for the reader.

10) L194. 'where': use 'in which' instead

Authors: Change applied.

11) L201-239 and Table1. stating $\mathrm{CH} 4: \mathrm{CO} 2$ ratios in $\mu \mathrm{mol} / \mathrm{mol}$ instead of $\mathrm{nmol} / \mathrm{mmol}$ would improve clarity.

Authors: Thank you for your suggestion. The units of the $\mathrm{CH} 4$ : $\mathrm{CO} 2$ ratios were changed accordingly throughout the whole manuscript.

12) L314-315. 'distinct differences in the patterns': redundant structure, could be simplified.

Authors: We reworded the sentence.

13) L318-324. This section could use some language editing for better flow. e.g. L306: 'the used growth substrates': The growth substrates used for this study... or similar.

Authors: This section was revised for a better flow.

14) L319-320. 'consist of various amounts': contain distinct amount of cellulose, [..], and other compounds.

Printer-friendly version

Authors: Changes applied.

Discussion paper

15) L320-321. structure in parenthesis: grammar 
Authors: Change applied.

16) L321-322. ... source signatures might_depend_on the metabolic pathways_used by_the fungal species_as well as_ the chemical composition of the substrate (or similar)

Authors: Change applied.

17) L323. Therefore, we suggest: remove this phrase. "The selected ..."

Authors: Change applied.

18) L326. Figure 5_compares__the_d13C-CH4 values.

Authors: Change applied.

19) L334. 'depending on the photosynthetic pathway (C3, C4, or CAM)'

Authors: Change applied.

Interactive comment on Biogeosciences Discuss., https://doi.org/10.5194/bg-2020-108, 2020. 